TAIWANESE JOURNAL OF MATHEMATICS

Vol. 12, No. 9, pp. 2341-2361, December 2008

This paper is available online at http://www.tjm.nsysu.edu.tw/

\title{
SPLITTING EXTRAPOLATIONS FOR SOLVING BOUNDARY INTEGRAL EQUATIONS OF MIXED BOUNDARY CONDITIONS ON POLYGONS BY MECHANICAL QUADRATURE METHODS
}

\author{
Jin Huang, Zi Cai Li*, Tao Lü and Rui Zhu
}

\begin{abstract}
To solve the boundary integral equations (BIE) of mixed boundary conditions, we propose the mechanical quadrature methods (MQM) using specific quadrature rule to deal with weakly singular integrals. Denote $h_{m}$ as the mesh width of a curved edge $\Gamma_{m}(m=1, \ldots, d)$ of polygons. Then the multivariate asymptotic expansions of solution errors are found to be $O\left(h^{3}\right)$, where $h=\max _{1 \leq m \leq d} h_{m}$. Hence, by using the splitting extrapolation methods (SEM), the high convergence rates as $O\left(h^{5}\right)$ can be achieved. Moreover, numerical examples are provided to support our theoretical analysis.
\end{abstract}

\section{INTRODUCTION}

In this paper we develop mechanical quadrature methods (MQM) and splitting extrapolation methods (SEM) for solving boundary integral equations (BIE) of the mixed boundary value problems:

$$
\begin{cases}\Delta u=0, & \text { in } \Omega, \\ \alpha_{m} u+\beta_{m} \frac{\partial u}{\partial n}=g, & \text { on } \Gamma_{m}, m=1, \ldots, d,\end{cases}
$$

where $\Omega \subset \Re^{2}$ is a curved polygon with the edges $\Gamma=\cup_{m=1}^{d} \Gamma_{m}$, and the angles between $\Gamma_{m}$ and $\Gamma_{m+1}$ are in $(0,2 \pi]$. We assume that $g$ is a piecewise continuous function on $\Gamma$, and $\alpha_{m}$ and $\beta_{m}$ are constants on $\Gamma_{m}$.

Received February 24, 2006, accepted June 5, 2007.

Communicated by W. W. Lin.

2000 Mathematics Subject Classification: 65R20, 45L10.

Key words and phrases: Mixed boundary condition, Polygon, Mechanical quadrature method, Splitting extrapolation, Boundary integral equation.

The first author was supported by National Science Foundation of China (10171073).

*Corresponding author. 
By Green's formula, Eq (1.1) is converted into BIE,

$$
-\int_{\Gamma} \frac{\partial u(x)}{\partial n_{x}} \log |y-x| d s_{x}+\int_{\Gamma} u(x) \frac{\partial}{\partial n_{x}} \log |y-x| d s_{x}=\theta(y) u(y),
$$

where $|y-x|^{2}=\left(y_{1}-x_{1}\right)^{2}+\left(y_{2}-x_{2}\right)^{2}$ and $\theta(y)$ is a constant dependent on $y \in \Gamma$. If one of $u(x)$ and $\frac{\partial u(x)}{\partial n_{x}}$ is given by the boundary conditions of (1.1), then the other can be solved by (1.2). Once both $\frac{\partial u(x)}{\partial n_{x}}$ and $u(x)(x \in \Gamma)$ are known, the solution $u(y)(y \in \Omega)$ can be calculated by

$$
u(y)=\frac{1}{2 \pi} \int_{\Gamma} u(x) \frac{\partial}{\partial n_{x}} \log |y-x| d s_{x}-\frac{1}{2 \pi} \int_{\Gamma} \frac{\partial u(x)}{\partial n_{x}} \log |y-x| d s_{x} .
$$

It is known $[4,9,17,24]$ that if all $\beta_{m}=0$ on $\Gamma_{m}(m=1, \ldots, d)$, then $(1.1)$ becomes a pure Dirichlet problem, and Eq (1.2) is the weakly singular BIE system of the first kind, whose solution exists and is unique as $C_{\Gamma} \neq 1$, where $C_{\Gamma}$ is the logarithmic capacity (i.e., the transfinite diameter). When $\Gamma$ is a circle, $C_{\Gamma}$ is just the diameter. If all $\alpha_{m}=0$ on $\Gamma_{m}(m=1, \ldots, d)$, then (1.1) becomes a pure Neumann problem, and Eq (1.2) is the weakly singular BIE system of the second kind, whose solution exists if and only if

$$
\sum_{m=1}^{d} \int_{\Gamma_{m}} g / \beta_{m} d s=0 .
$$

Moreover, if $\alpha_{m} \beta_{m}>0$ on $\Gamma_{m}(m=1, \ldots, d)$, or both the Dirichlet and the Neuman boundary conditions are assigned in different $\Gamma_{m}(m=1, \ldots, d)$, then (1.1) has a unique solution, and (1.2) is the weakly singular mixed BIE system.

Galerkin and collocation methods [1, 2, 5, 18, 19, 25] are often applied to solve BIEs, but they are complicated for calculating discrete matrix. Using Galerkin methods, Rüde and Zhou [18] established multi-parameter extrapolation methods for BIE system of the second kind on polygonal domains. Assuming that $\Omega$ is a bounded and simply connected open region with a smooth boundary $\Gamma$, and the inverse matrix of discrete equations exists and is uniformly bounded, $\mathrm{Xu}$ and Zhao [24] established an extrapolation method for solving BIE from the boundary value problem of the third kind. Assuming that $\Omega$ is a bounded simply-connected domain, and that $\Gamma_{m}(m=1, \ldots, d)$ are straight lines or circular arcs, Symm [4] applied collocation methods to solve (1.2). In this paper, firstly, taking $h_{m}$ as the mesh width of a curved edge $\Gamma_{m}(m=1, \ldots, d)$ of the polygon and using quadrature rules for weakly singular integrals, we construct the mechanical quadrature methods (MQM) for solving BIE of mixed boundary conditions. Secondly, we obtain the multivariate asymptotic expansions of the solutions. Hence, once discrete equations with coarse grids of $\Gamma_{m}(m=1, \ldots, d)$ are solved in parallel, the accuracy of 
approximate solutions can be greatly improved by the splitting extrapolation methods (SEM). Finally, a posteriori error estimates as self-adaptive algorithms are derived. Obviously, the mechanical quadrature methods (MQM) are very simple, because of no needs of calculating any integrals for discrete matrix. In this paper, we will provide their error analysis.

SEM $[11,13,14,16]$ based on multivariate asymptotic expansions of the errors is a very effective parallel algorithm, because it possesses a high order of accuracy, good stability and almost optimal computational complexity. Since Lin and Lü published the first SEM paper [14] in 1983, SEM has been applied to many problems, such as the multidimensional numerical integrations [11, 13], finite differential methods and finite element methods [13]. In the paper, MQM and SEM are also applied to solve BIE of mixed boundary conditions on polygons.

This paper is organized as follows. In Section 2 we discuss the weakly singular BIE. In Section 3, we establish MQM and prove its convergence. In Section 4, the multivariate asymptotic expansions of errors are derived, and the SEM is described. In Section 5, numerical examples are provided.

\section{Weakly Singular BIE}

Define boundary integral operators on $\Gamma_{m}$,

$$
\left(A_{q m} w_{m}\right)(y)=-\frac{1}{2 \pi} \int_{\Gamma_{m}} w_{m}(x) \log |y-x| d s_{x}, y \in \Gamma_{q}, m, q=1, \ldots, d,
$$

and

$$
\left(K_{q m} w_{m}\right)(y)=-\frac{1}{2 \pi} \int_{\Gamma_{m}} w_{m}(x) \frac{\partial}{\partial n_{x}} \log |y-x| d s_{x}, y \in \Gamma_{q}, m, q=1, \ldots, d
$$

where

$$
w_{m}(x)=\left\{\begin{array}{c}
\frac{\partial u(x)}{\partial n}, x \in \Gamma_{m}, \text { if } \beta_{m}=0 \text { on } \Gamma_{m}, \\
u(x), x \in \Gamma_{m}, \text { if } \beta_{m} \neq 0 \text { on } \Gamma_{m} .
\end{array}\right.
$$

Then (1.2) can be converted into a matrix operator equation

$$
\beta \theta W+\alpha A W+\beta K W=F,
$$

where $\theta=\operatorname{diag}\left(\theta_{1}(y), \ldots, \theta_{d}(y)\right), A=\left[A_{q m}\right]_{q, m=1}^{d}, K=\left[K_{q m}\right]_{q, m=1}^{d}, W=\left(w_{1}(x)\right.$, $\left.\ldots, w_{d}(x)\right)^{T}, \alpha=\operatorname{diag}\left(\alpha_{1}(y), \ldots, \alpha_{d}(y)\right), \beta=\operatorname{diag}\left(\beta_{1}(y), \ldots, \beta_{d}(y)\right)$ and $F=\left(f_{1}(y)\right.$, 
$\left.\ldots, f_{d}(y)\right)^{T}$ with

$$
f_{q}(y)=\left\{\begin{array}{l}
\theta_{q}(y) g_{q}(y)+\sum_{m=1}^{d}\left(K_{q m} g_{m}\right)(y), \text { if } \beta_{q}=0 \\
\sum_{m=1}^{d}\left(A_{q m} g_{m}\right)(y), \text { if } \beta_{q} \neq 0, q=1, \ldots, m
\end{array}\right.
$$

Assume that $\Gamma_{m}$ can be described by the parameter mapping

$$
x_{m}(s)=\left(x_{m 1}(s), x_{m 2}(s)\right):\left[0, T_{m}\right] \rightarrow \Gamma_{m}
$$

with $\left|x_{m}^{\prime}(s)\right|^{2}=\left|x_{m 1}^{\prime}(s)\right|^{2}+\left|x_{m 2}^{\prime}(s)\right|^{2}>0$, where $T_{m}$ is the measurable length of $\Gamma_{m}$. Using the trigonometric transformation [20]

$$
s=T_{m} \varphi_{p}(t):[0,1] \rightarrow\left[0, T_{m}\right], p \in N,
$$

with

$$
\varphi_{p}(t)=\vartheta_{p}(t) / \vartheta_{p}(1) \text { and } \vartheta_{p}(t)=\int_{0}^{t}(\sin \pi t)^{p} d s,
$$

the operators (2.1) and (2.2) are also converted into integral operators on $[0,1]$

$$
\left(\bar{A}_{q 0} \bar{w}_{q}\right)(t)=-\frac{1}{\pi} \int_{0}^{1} z_{q}(t) \ln \left|2 e^{-1 / 2} \sin \pi(t-\tau)\right| \bar{w}_{q}(\tau) d \tau, t \in[0,1),
$$

$$
\begin{gathered}
\left(\bar{A}_{0 q} \bar{w}_{q}\right)(t)=-\frac{1}{\pi} \int_{0}^{1} z_{q}(t) \ln \left|\frac{\bar{x}_{q}(t)-\bar{x}_{q}(\tau)}{2 e^{-1 / 2} \sin \pi(t-\tau)}\right| \bar{w}_{q}(\tau) d \tau, t \in[0,1), \\
\left(\bar{A}_{q m} \bar{w}_{m}\right)(t)=-\frac{1}{\pi} \int_{0}^{1} z_{q}(t) \ln \left|\bar{x}_{q}(t)-\bar{x}_{m}(\tau)\right| \bar{w}_{m}(\tau) d \tau, q \neq m, t \in[0,1),
\end{gathered}
$$

and

$$
\left(\bar{K}_{q m} \bar{w}_{m}\right)(t)=-\frac{1}{\pi} \int_{0}^{1} z_{q}(t) \tilde{k}_{q m}(t, \tau) \bar{w}_{m}(\tau) d \tau, t \in[0,1)
$$

where $\bar{w}_{m}(t)=w_{m}\left(\bar{x}_{m}(t)\right) T_{q} \varphi_{p}^{\prime}(t)\left|\bar{x}_{m}^{\prime}(t)\right|$ and $\bar{x}_{m}(t)=x_{m}\left(T_{m} \varphi_{p}(t)\right)$. In (2.7)(2.10) $\left|\bar{x}_{q}(t)-\bar{x}_{m}(\tau)\right|^{2}=\left(\bar{x}_{q 1}(t)-\bar{x}_{m 1}(\tau)\right)^{2}+\left(\bar{x}_{q 2}(t)-\bar{x}_{m 2}(\tau)\right)^{2}$ and

$$
z_{q}(t)= \begin{cases}1, & \text { as } \beta_{q}=0, \\ T_{q} \varphi_{p}^{\prime}(t)\left|\bar{x}_{q}^{\prime}(t)\right|, & \text { as } \beta_{q} \neq 0 .\end{cases}
$$


In (2.10) $\tilde{k}_{q m}(t, \tau)$ results from $\frac{\partial}{\partial n_{x}} \log |y-x|$ under the parameter mapping (2.4) and the trigonometric transformation (2.5). Then Eq (2.3) can be expressed by

$$
\left(\beta E+\alpha \bar{A}_{0}+\alpha \bar{A}_{1}+\alpha \bar{A}_{2}+\beta \bar{K}\right) \bar{W}=\bar{F},
$$

where $\bar{A}_{0}=\operatorname{diag}\left(\bar{A}_{10}, \ldots, \bar{A}_{d 0}\right), \bar{A}_{1}=\operatorname{diag}\left(\bar{A}_{01}, \ldots, \bar{A}_{0 d}\right), \bar{A}_{2}=\left[\bar{A}_{q m}\right]_{q, m=1}^{d}$, and $\bar{K}=\left[\bar{K}_{q m}\right]_{q, m=1}^{d}$ are matrix operators. In (2.12) $\bar{W}=\left(\bar{w}_{1}(t), \ldots, \bar{w}_{d}(t)\right)^{T}$ and $\bar{F}=\left(\bar{f}_{1}(t), \ldots, \bar{f}_{d}(t)\right)^{T}$ with

$$
\bar{f}_{q}(t)=\left\{\begin{array}{l}
2 g_{q}\left(\bar{x}_{q}(t)\right)+\sum_{m=1}^{d}\left(\bar{K}_{q m} \bar{g}_{m}\right)(t), \text { as } \beta_{q}=0, \\
\left(\left(\bar{A}_{q 0}+\bar{A}_{0 q}\right) \bar{g}_{q}\right)(t)+\sum_{m=1}^{d}\left(\bar{A}_{q m} \bar{g}_{m}\right)(t), \text { as } \beta_{q} \neq 0
\end{array}\right.
$$

where $\bar{g}_{m}(t)=g_{m}\left(\bar{x}_{m}(t)\right) T_{q} \varphi_{p}^{\prime}(t)\left|\bar{x}_{q}^{\prime}(t)\right|$. Let $a_{q 0}(t, \tau), a_{0 q}(t, \tau), a_{q m}(t, \tau)$ and $k_{q m}(t, \tau)$ be the kernels of the integral operators $\bar{A}_{q 0}, \bar{A}_{0 q}, \bar{A}_{q m}$ and $\bar{K}_{q m}$, respectively. Then the following results hold.

(1) The solutions of (2.3) are equivalent to those of (2.12).

(2) $a_{q 0}(t, \tau)$ is a logarithmically singular function on $t \in[0,1]$ and $\tau \in[0,1]$.

(3) $a_{0 q}(t, \tau)$ is a continuous function $[11,15,25]$ on $t \in[0,1]$ and $\tau \in[0,1]$.

(4) For $\Gamma_{q} \cap \Gamma_{m}=\emptyset$ (i.e., $|q-m| \neq 1$, or $\left.d-1\right), a_{q m}(t, \tau)$ and $k_{q m}(t, \tau)$ are continuous functions $[1,5,11,22]$ on $t \in[0,1]$ and $\tau \in[0,1]$.

(5) Although $\frac{\partial u_{q}(x)}{\partial n}$ at an angular point $x=\Gamma_{q} \cap \Gamma_{m}$ has the singularity, $\bar{w}_{q}(t)$ $(t \in[0,1])$ is a smooth function $[11,21,22]$ under (2.4)-(6).

Lemma 1. If $\Gamma_{q} \cap \Gamma_{m} \neq \emptyset$ (i.e., $|q-m|=1$, or $d-1$ ) and $p \geq 3$, then (1) for $\beta_{q}=0, \tilde{a}_{q m}(t, \tau)\left(=\sin ^{p}(\pi t) a_{q m}(t, \tau)\right)$ and $\frac{\partial^{n}}{\partial t^{n}} \tilde{a}_{q m}(t, \tau)(n=1,2,3)$ are continuous on $[0,1]^{2}$, and (2) for $\beta_{q} \neq 0, a_{q m}(t, \tau)$ and $k_{q m}(t, \tau)$ are continuous on $[0,1]^{2}$.

Proof. Without loss of generality, we assume that the origin $(0,0)=\Gamma_{m} \cap \Gamma_{q}$ of coordinates is a vertex with an interior angle $\theta_{q}$.

(1) Firstly, for $\theta_{q} \in(0, \pi) \cup(\pi, 2 \pi)$, using the cosine theorem ${ }^{[7]}$ and (2.9) we get

$$
\begin{aligned}
& \tilde{a}_{q m}(t, \tau)=\sin ^{p}(\pi t) a_{q m}(t, \tau)=-(2 \pi)^{-1} \sin ^{p}(\pi t) \ln \left[a_{0}^{2}(t)+a_{1}^{2}(\tau)\right. \\
& \left.-2 a_{0}(t) a_{1}(\tau) \cos \theta_{q}\right]=-(2 \pi)^{-1} \sin ^{p}(\pi t)\left\{\ln \left(a_{0}^{2}(t)+a_{1}^{2}(\tau)\right)\right. \\
& \left.-\ln \left[1-2 a_{0}(t) a_{1}(\tau) \cos \theta_{q} /\left(a_{0}^{2}(t)+a_{1}^{2}(\tau)\right)\right]\right\}
\end{aligned}
$$


where $a_{0}(t)=\left|x_{m}\left(T_{m} \varphi_{p}(t)\right)\right|$ and $a_{1}(\tau)=\left|x_{q}\left(T_{q} \varphi_{p}(\tau)\right)\right|$. Also without loss of generality, we assume $a_{0}(0)=a_{1}(0)=0$. Since

$$
\left|2 a_{0}(t) a_{1}(\tau) \cos \theta_{q} /\left(a_{0}^{2}(t)+a_{1}^{2}(\tau)\right)\right| \leq\left|\cos \theta_{q}\right|<1,
$$

$\tilde{a}_{q m}(t, \tau)$ will be continuous if we can prove that $a(t, \tau)=\sin ^{p}(\pi t) \ln \left(a_{0}^{2}(t)+\right.$ $\left.a_{1}^{2}(\tau)\right)$ is a bounded function on $[0,1]^{2}$. In fact, from $\left.\varphi_{p}^{(j)}(t)\right|_{t=0,1}=0(j=1, \ldots, p)$, we easily get $\left.a_{i}^{(j)}(t)\right|_{t=0, t=1}=0(j=1, \ldots, p, i=0,1)$. Thus we need only prove that $a(t, \tau)$ is bounded on $[\varepsilon / 2, \varepsilon]^{2}, \forall \varepsilon>0$. For $(t, \tau) \in[\varepsilon / 2, \varepsilon]^{2}$, it always holds that

$$
|a(t, \tau)|=O\left(\varepsilon^{p}|\ln \varepsilon|\right) \rightarrow 0, \text { as } \varepsilon \rightarrow 0,
$$

which means that $a(t, \tau)$ is bounded. Similarly, from

$$
\left|\frac{\partial}{\partial \tau} a(t, \tau)\right|=\left|\sin ^{p}(\pi t) \frac{2 a_{1}(\tau) \bar{x}_{q}^{\prime}(\tau) \varphi_{p}^{\prime}(\tau)}{\left(a_{0}^{2}(t)+a_{1}^{2}(\tau)\right)}\right|=O\left(\varepsilon^{p}\right), \text { as } \varepsilon \rightarrow 0
$$

and

$$
\left|\frac{\partial^{2}}{\partial \tau^{2}} a(t, \tau)\right|=O\left(\varepsilon^{p-1}\right),\left|\frac{\partial^{3}}{\partial \tau^{3}} a(t, \tau)\right|=O\left(\varepsilon^{p-2}\right), \text { as } \varepsilon \rightarrow 0, p \geq 3,
$$

we can prove that $\frac{\partial^{n}}{\partial t^{n}} a(t, \tau)(n=1,2,3)$ are continuous on $[0,1]^{2}$.

Secondly, for $\theta_{q}=\pi$, we have

$$
\tilde{a}_{q m}(t, \tau)=-\sin ^{p}(\pi t)\left(\ln \left[a_{0}(t)+a_{1}(\tau)\right]\right) / \pi .
$$

Following the above proof, we can prove that $\frac{\partial^{n}}{\partial t^{n}} \tilde{a}_{q m}(t, \tau)(n=1,2,3)$ are continuous on $[0,1]^{2}$ for $p \geq 3$.

(2) Firstly, for $\theta_{q} \in(0, \pi) \cup(\pi, 2 \pi)$, we get

$$
a_{q m}(t, \tau)=-\bar{z}_{q} \sin ^{p}(\pi t) \ln \left[a_{0}^{2}(t)+a_{1}^{2}(\tau)-2 a_{0}(t) a_{1}(\tau) \cos \theta_{q}\right],
$$

where $\bar{z}_{q}=T_{q}\left|\bar{x}_{q}^{\prime}(t)\right| / \vartheta_{p}(1) /(2 \pi)$. For $\theta_{q}=\pi$,

$$
a_{q m}(t, \tau)=-2 \bar{z}_{q} \sin ^{p}(\pi t) \ln \left[a_{0}(t)+a_{1}(\tau)\right] .
$$

Hence, for $(t, \tau) \in[\varepsilon / 2, \varepsilon]^{2}$ we have

$$
\left|a_{q m}(t, \tau)\right|=O\left(\varepsilon^{p}|\ln \varepsilon|\right) \rightarrow 0, \text { as } \varepsilon \rightarrow 0 .
$$

Secondly, using the cosine theorem ${ }^{[7]}$ and (2.10) we get

$$
k_{q m}(t, \tau)=\frac{-2 \bar{z}_{q} \sin ^{p}(\pi t) \tilde{k}(t, \tau)}{a_{0}^{2}(t)+a_{1}^{2}(\tau)-2 a_{0}(t) a_{1}(\tau) \cos \theta_{q}}, \text { for } \theta_{q} \in(0, \pi) \cup(\pi, 2 \pi),
$$


where $\tilde{k}(t, \tau)=\left(x_{q 2}^{\prime}\left(T_{q} \varphi_{p}(\tau)\right)\left(\bar{x}_{m 1}(\tau)-\bar{x}_{q 1}(t)\right)-x_{q 1}^{\prime}\left(T_{q} \varphi_{p}(\tau)\right)\left(\bar{x}_{m 2}(\tau)-\bar{x}_{q 2}(t)\right)\right)$, and

$$
k_{q m}(t, \tau)=-2 \bar{z}_{q} \frac{\sin ^{p}(\pi t) \tilde{k}(t, \tau)}{\left|a_{0}(t)+a_{1}(\tau)\right|^{2}}, \text { for } \theta_{q}=\pi .
$$

Hence, for $(t, \tau) \in[\varepsilon / 2, \varepsilon]^{2}$ we have

$$
|k(t, \tau)|=O(\varepsilon) \rightarrow 0, \text { as } \varepsilon \rightarrow 0 .
$$

Consequently, we can conclude that $a_{q m}(t, \tau)$ and $k_{q m}(t, \tau)$ are continuous on $[0,1]^{2}$.

\section{Mechanical Quadrature Method}

Let $h_{m}=1 / n_{m}\left(n_{m} \in N, m=1, \ldots, d\right)$ be mesh widths and $t_{j}=\tau_{j}=$ $(j-1 / 2) h_{m}\left(j=1, \ldots, n_{m}\right)$ be nodes. For an integral operator $D$ with continuous kernel as $\bar{A}_{0 q}, \bar{A}_{q m}$ or $\bar{K}_{q m}$, by the midpoint or the trapezoidal rule, we construct the Nyström approximation [8]

$$
\left(D^{h} \bar{w}_{m}\right)(t)=h_{m} \sum_{j=1}^{n_{m}} d\left(t, \tau_{j}\right) \bar{w}_{m}\left(\tau_{j}\right), t \in[0,1], q, m=1, \ldots, d,
$$

which has the following error bounds $[8,21]$

$$
\left(D \bar{w}_{m}\right)(t)-\left(D^{h} \bar{w}_{m}\right)(t)=O\left(h_{m}^{2 l}\right), l \in N .
$$

For the weakly singular operators $\bar{A}_{q 0}$, by the quadrature formula [20], we can also construct the Fredholm approximation,

$$
\begin{aligned}
& \left(\bar{A}_{q 0}^{h} \bar{w}_{q}\right)\left(t_{i}\right) \\
= & -z_{q}\left(t_{i}\right) h_{q}\left\{\sum_{j=1, t \neq \tau_{j}}^{n_{m}} \ln \left|2 e^{-1 / 2} \sin \pi\left(t_{i}-\tau_{j}\right) z_{q}\left(\tau_{j}\right)\right| \bar{w}_{q}\left(\tau_{j}\right)\right\} / \pi \\
& -h_{q}|\ln | 2 \pi e^{-1 / 2} h_{q} /(2 \pi) \mid z_{q}\left(t_{i}\right) \bar{w}_{q}\left(t_{i}\right) / \pi, i=1, \ldots, n_{q},
\end{aligned}
$$

which has the following error bounds $[20,21]$

$$
\begin{aligned}
& \left(\bar{A}_{q 0}^{h} \bar{w}_{q}\right)\left(t_{i}\right)-\left(\bar{A}_{q 0} \bar{w}_{q}\right)\left(t_{i}\right) \\
= & \frac{-2}{\pi} \sum_{\mu=1}^{2 l-1} \frac{\zeta^{\prime}(-2 \mu)}{(2 \mu) !}\left[z_{q}\left(t_{i}\right) \bar{w}_{q}\left(t_{i}\right)\right]^{(2 \mu)} h_{q}^{2 \mu+1}+O\left(h_{q}^{2 l}\right),
\end{aligned}
$$


where $\zeta^{\prime}(t)$ is the derivative of the Riemann Zeta function.

Consider the approximate equations of (2.12)

$$
\left(\beta E^{h}+\alpha \bar{A}_{0}^{h}+\alpha \bar{A}_{1}^{h}+\alpha \bar{A}_{2}^{h}+\beta \bar{K}^{h}\right) \bar{W}^{h}=\bar{F}^{h},
$$

where $\bar{W}^{h}=\left(\bar{W}_{1}^{h}, \ldots, \bar{W}_{d}^{h}\right)^{T}, \bar{W}_{m}^{h}=\left(\bar{w}_{m}\left(t_{1}\right), \ldots, \bar{w}_{m}\left(t_{n_{m}}\right)\right)^{T}, \bar{A}_{0}^{h}=\operatorname{diag}\left(\bar{A}_{10}^{h}, \ldots\right.$, $\left.\bar{A}_{d 0}^{h}\right), \bar{A}_{q 0}^{h}=\left[a_{q 0}\left(t_{j}, \tau_{i}\right)\right]_{j, i=1}^{n_{q}}, \bar{A}_{1}^{h}=\operatorname{diag}\left(\bar{A}_{01}^{h}, \ldots, \bar{A}_{0 d}^{h}\right), \bar{A}_{0 q}^{h}=\left[a_{0 q}\left(t_{j}, \tau_{i}\right)\right]_{j, i=1}^{n_{q}}$, $\bar{A}_{2}^{h}=\left[\bar{A}_{q m}^{h}\right]_{q, m=1}^{d}, \bar{A}_{q m}^{h}=\left[a_{q m}\left(t_{j}, \tau_{i}\right)\right]_{j, i=1}^{n_{q}, n_{m}}, \bar{K}^{h}=\left[\bar{K}_{q m}^{h}\right]_{q, m=1}^{d}, \bar{K}_{q m}^{h}=\left[k_{q m}\left(t_{j}\right.\right.$, $\left.\left.\tau_{i}\right)\right]_{j, i=1}^{n_{q}, n_{m}}, \bar{F}^{h}=\left(\bar{F}_{1}^{h}, \ldots, \bar{F}_{d}^{h}\right)^{T}, \bar{F}_{q}^{h}=\left(\bar{f}_{q 1}^{h}, \ldots, \bar{f}_{q n_{q}}^{h}\right)^{T}$ and

$$
\bar{f}_{q j}^{h}=\left\{\begin{array}{l}
2 \bar{g}_{q}\left(t_{j}\right)+\sum_{m=1}^{d}\left(\bar{K}_{q m}^{h} \bar{g}_{m}\right)\left(t_{j}\right), \text { as } \beta_{q}=0, \\
\left(\left(\bar{A}_{q 0}^{h}+\bar{A}_{0 q}^{h}\right) \bar{g}_{q}\right)\left(t_{j}\right)+\sum_{m=1}^{d}\left(\bar{A}_{q m}^{h} \bar{g}_{m}\right)\left(t_{j}\right), \text { as } \beta_{q} \neq 0 .
\end{array}\right.
$$

Obviously, (3.6) is a linear equation system with $n$-unknowns, where $n=n_{1}+$ $\ldots+n_{d}$. Once $\bar{W}^{h}$ is solved by (3.6), the solution $u(y)(y \in \Omega)$ can be computed by

$$
\begin{aligned}
u(y)= & \frac{1}{2 \pi} \sum_{m=1}^{d} \sum_{i=1}^{n_{m}} h_{m}\left[u_{m}\left(\bar{x}\left(t_{i}\right)\right) \hat{k}_{m}(y, x)\right. \\
& \left.-\frac{\partial u\left(\bar{x}\left(t_{i}\right)\right)}{\partial n_{x}} \ln \left|y-\bar{x}_{m}\left(t_{i}\right)\right|\right]\left|\bar{x}_{m}^{\prime}\left(t_{i}\right)\right|,
\end{aligned}
$$

where

$$
\hat{k}_{m}(y, x)=\frac{\bar{x}_{m 2}^{\prime}\left(t_{i}\right)\left(\bar{x}_{m 1}\left(t_{i}\right)-y_{1}\right)-\bar{x}_{m 1}^{\prime}\left(t_{i}\right)\left(\bar{x}_{m 2}\left(t_{i}\right)-y_{2}\right)}{\left[\left(\bar{x}_{m 1}\left(t_{i}\right)-y_{1}\right)^{2}+\left(\bar{x}_{m 2}\left(t_{i}\right)-y_{2}\right)^{2}\right]\left|\bar{x}_{m}^{\prime}\left(t_{i}\right)\right|} .
$$

To show a unique solution existing for (3.6), we first prove that the operator $\beta_{m} E^{h}+\alpha_{m} \bar{A}_{m 0}^{h}$ is invertible. Define $\mathcal{C}_{m}=\operatorname{diag}\left(z_{m}\left(t_{1}\right), \ldots, z_{m}\left(t_{n_{m}}\right)\right)$ with $z_{m}\left(t_{j}\right)>0\left(j=1, \ldots, n_{m}\right)$ and $\mathcal{D}_{m}=-h_{m} / \pi$ circular $\left(\ln \left(e^{-1 / 2} h_{m}\right), \ln \left(2 e^{-1 / 2}\right.\right.$ $\left.\left.\sin \left(\pi h_{m}\right)\right), \ldots, \ln \left(2 e^{-1 / 2} \sin \left(\left(n_{m}-1\right) \pi h_{m}\right)\right)\right)$. From (3.3), we get $\bar{A}_{m 0}^{h}=\mathcal{C}_{m} \mathcal{D}_{m}$.

Lemma 2. The eigenvalues $\lambda_{k}\left(k=1, \ldots, n_{m}\right)$ of $\mathcal{D}_{m}$ are positive, and there exists a positive constant $c$ such that $\lambda_{k}>c$ for $n_{m}<4$, or $\lambda_{k}>1 /\left(2 \pi n_{m}\right)$ for $n_{m} \geq 4$.

Proof. Since $\mathcal{D}_{m}$ is a symmetric circulant matrix, we have $\lambda_{k}=F\left(\varepsilon_{k}\right)$ with $F(z)=-h_{m}\left[\ln \left|h e^{-1 / 2}\right|+\sum_{j=1}^{n_{m}-1} z^{j} \ln \left|2 e^{-1 / 2} \sin \left(j \pi / n_{m}\right)\right|\right]$, and $\varepsilon_{k}=\exp \left(2 \pi k i / n_{m}\right)$. 
If $n_{m}<4$, then $\lambda_{k}>c$ can be easily verified by direct calculations. On the other hand, if $n_{m} \geq 4$, then $\lambda_{k}$ is estimated in two steps:

Step 1. Consider $k=0$. Let

$$
\begin{aligned}
& \lambda_{0}^{\prime}=\ln \left|h_{m} e^{-1 / 2}\right|+\sum_{j=1}^{n_{m}-1} \ln \left|2 e^{-1 / 2} \sin \left(j \pi / n_{m}\right)\right| \\
& =-n_{m} / 2-\ln n_{m}+\ln \left|2^{n_{m}-1} \prod_{j=1}^{n_{m}-1} \sin \left(j \pi / n_{m}\right)\right| .
\end{aligned}
$$

We will discuss the following two cases.

Case 1. For $n_{m}=2 l-1$, by the inequality

$$
2 x / \pi<\sin x<x, \text { if } 0<x<\pi / 2,
$$

we have

$$
\frac{2^{2 l-2}[(l-1) !]^{2}}{(2 l-1)^{2 l-2}}<\prod_{j=1}^{l-1} \sin ^{2} \frac{j \pi}{2 l-1}=\prod_{j=1}^{n_{m}-1} \sin \frac{j \pi}{n_{m}}<\frac{\pi^{2 l-2}[(l-1) !]^{2}}{(2 l-1)^{2 l-2}} .
$$

Using Stirling's rule $n !=\sqrt{2 \pi n}(n / e)^{n} \exp (\theta /(12 n))(0<\theta<1)$, we obtain

$$
\frac{2^{2 l-2}[(l-1) !]^{2}}{(2 l-1)^{2 l-2}}>2 \pi e^{2-2 l}(l-1)(1-1 / l)^{2 l-2} e^{\theta /[6(l-1)]}
$$

and

$$
\frac{\pi^{2 l-2}[(l-1) !]^{2}}{(2 l-1)^{2 l-2}}<2 \pi\left(\frac{\pi}{2 e}\right)^{2 l-2}(l-1) e^{\theta /[6(l-1)]}=1 / B .
$$

Also since $\ln B<\ln \left|2^{n_{m}-1} \prod_{j=1}^{n_{m}-1} \sin \left(j \pi / n_{m}\right)\right|^{-1}$, we get

$$
\lambda_{0}=-\lambda_{0}^{\prime} /\left(\pi n_{m}\right)>[3 / 2-\ln \pi-1 /(2 l-1)-1 /[6(2 l-1)(l-1)] / \pi,
$$

which implies that $\lambda_{0}>17 /(150 \pi)$ for $l \geq 3$.

Case 2. For $n_{m}=2 l$, from $0<(l-1) /(2 l)<1 / 2$ and (3.9), we obtain

$$
\frac{[(l-1) !]^{2}}{l^{2 l-2}}<\prod_{j=1}^{l-1} \sin ^{2} \frac{j \pi}{2 l}=\prod_{j=1}^{n_{m}-1} \sin \frac{j \pi}{n_{m}}<\frac{\pi^{2 l-2}[(l-1) !]^{2}}{(2 l)^{2 l-2}} .
$$


Using

$$
\frac{\pi^{2 l-2}[(l-1) !]^{2}}{(2 l)^{2 l-2}}=2 l \pi\left(\frac{\pi}{2}\right)^{2 l-2} e^{(-2 l+\theta / 6 l)}
$$

and the above inequality, we have

$$
\begin{gathered}
\lambda_{0}=-\lambda_{0}^{\prime} /\left(\pi n_{m}\right)=\left[1 / 2+1 / n_{m} \ln n_{m}+1 / n_{m} \ln \mid 2^{n_{m}-1}\right. \\
\left.\left.\prod_{j=1}^{n_{m}-1} \sin \left(j \pi / n_{m}\right)\right|^{-1}\right] / \pi>[3 / 2-\ln \pi] / \pi
\end{gathered}
$$

which implies that $\lambda_{0}>c>0$ as $n_{m} \geq 4$.

Step 2. To estimate $\lambda_{k}, k=1, \ldots, n_{m}-1$, we write

$$
\begin{aligned}
\lambda_{k}^{\prime} & =\ln \left|e^{-1 / 2} / n_{m}\right|+\sum_{j=1}^{n_{m}-1} \cos \left(2 k j \pi / n_{m}\right) \ln \left|2 e^{-1 / 2} \sin \left(j \pi / n_{m}\right)\right| \\
& =-\ln n_{m}+\sum_{j=1}^{n_{m}-1} \cos \left(2 k j \pi / n_{m}\right) \ln \left|2 \sin \left(j \pi / n_{m}\right)\right| .
\end{aligned}
$$

Using the expansions of the $\psi$-special function [7]

$$
\psi(k / n)=-\gamma-\ln n-\pi / 2 \cot (k \pi / n)+\sum_{j=1}^{n} \cos (2 k j \pi / n) \ln |2 \sin (j \pi / n)|
$$

and

$$
\psi(z)=-\gamma-1 / z+z \sum_{j=1}^{\infty} 1 /[j(j+z)]
$$

we obtain

$$
\begin{aligned}
& \sum_{j=1}^{n_{m}-1} \cos \left(2 k j \pi / n_{m}\right) \ln \left|2 \sin \left(j \pi / n_{m}\right)\right| \\
= & \ln n_{m}+\pi / 2 \cot \left(k \pi / n_{m}\right)-n_{m} / k+k / n_{m} \sum_{j=1}^{\infty}\left[j\left(j+k / n_{m}\right)\right]^{-1}
\end{aligned}
$$

and

$$
\begin{aligned}
\lambda_{k}^{\prime} & =\pi / 2 \cot \left(k \pi / n_{m}\right)-n_{m} / k+k / n_{m} \sum_{j=1}^{\infty}\left[j\left(j+k / n_{m}\right)\right]^{-1}, \\
& 1 \leq k \leq n_{m}-1,
\end{aligned}
$$


where $\gamma$ is the Euler's constant. Substituting

$$
\begin{aligned}
\cot \left(k \pi / n_{m}\right)= & n_{m} /(k \pi)-k \pi /\left(3 n_{m}\right)-1 / 45\left(k \pi / n_{m}\right)^{3}-\ldots \\
& -2^{2 j} B_{j} /(2 j) !\left(k \pi / n_{m}\right)^{2 j-1}-\ldots
\end{aligned}
$$

into (3.11), we have

$$
\begin{aligned}
\lambda_{k}^{\prime}= & -n_{m} /(2 k)-k \pi^{2} /\left(6 n_{m}\right)-\ldots-2^{2 j+1} B_{j} /(2 j) !\left(k \pi / n_{m}\right)^{2 j-1} \pi \\
& -\ldots+k / n_{m} \sum_{j=1}^{\infty}\left[j\left(j+k / n_{m}\right)\right]^{-1}
\end{aligned}
$$

and

$$
\begin{aligned}
\lambda_{k}= & \left\{1 /(2 k)+k \pi^{2} /\left(6 n_{m}^{2}\right)+\ldots+2^{2 j+1} B_{j} /(2 j) !\left(k \pi / n_{m}\right)^{2 j-1} \pi / n_{m}\right. \\
& \left.+\cdots-k / n_{m}^{2} \sum_{j=1}^{\infty}\left[j\left(j+k / n_{m}\right)\right]^{-1}\right\} / \pi,
\end{aligned}
$$

where $B_{j}$ is the Bernoulli number. Moreover, since

$k \pi^{2} /\left(6 n_{m}^{2}\right)-k / n_{m}^{2} \sum_{j=1}^{\infty}\left[j\left(j+k / n_{m}\right)\right]^{-1}>k / n_{m}^{2}\left\{\sum_{j=1}^{\infty}\left[j^{-2}-(j(j+1 / 2))^{-1}\right]\right\}>0$,

we obtain

$$
\lambda_{k}>1 /(2 \pi k)+1 / 90\left(k / n_{m}\right)^{3} / n_{m}+\ldots>1 /(2 \pi k)>1 /\left(2 \pi n_{m}\right) .
$$

From the results of Step 1 and Step 2, the proof of Lemma 2 is completed.

From Lemma 2 we have the following corollary.

Corollary 1. (1) The conditional number of $\mathcal{D}_{m}$ is $O\left(n_{m}\right)$. (2) $\mathcal{D}_{m}$ is invertible and $\left\|\left(\mathcal{D}_{m}\right)^{-1}\right\|=O\left(n_{m}\right)$, where $\|\cdot\|$ denotes the spectral norm.

Lemma 3. (1) If $\beta_{m}=0$, then $\alpha_{m} A_{m 0}^{h}$ is invertible and

$$
\left\|\left(\alpha_{m} A_{m 0}^{h}\right)^{-1}\right\|=O\left(n_{m}\right) .
$$

(2) If $\beta_{m} \neq 0$ and $\beta_{m} \alpha_{m} \geq 0$, then $\beta_{m} E^{h}+\alpha_{m} \bar{A}_{m 0}^{h}$ is invertible and $\left(\beta_{m} E^{h}+\right.$ $\left.\alpha_{m} \bar{A}_{m 0}^{h}\right)^{-1}$ is uniformly bounded, i.e.,

$$
\left\|\left(\beta_{m} E^{h}+\alpha_{m} \bar{A}_{m 0}^{h}\right)^{-1}\right\| \leq\left|\beta_{m}^{-1}\right|
$$

Proof. (1) If $\beta_{m}=0$, then $\alpha_{m} A_{m 0}^{h}=\alpha_{m} \mathcal{D}_{m}$ and (3.13) holds. 
(2) If $\beta_{m} \neq 0$ and $\beta_{m} \alpha_{m} \geq 0$, from $A_{m 0}^{h}=\mathcal{C}_{m} \mathcal{D}_{m}$ and [6,10], we obtain $\lambda\left(\bar{A}_{m 0}^{h}\right) \geq \lambda_{\min }\left(\bar{A}_{m 0}^{h}\right)>0$ and $\left|\lambda_{i}\left(\beta_{m} E^{h}+\alpha_{m} \bar{A}_{m 0}^{h}\right)\right|=\left|\beta_{m}+\alpha_{m} \lambda\left(\bar{A}_{m 0}^{h}\right)\right|$ $\geq\left|\beta_{m}\right|$. Then (3.13) holds.

Corollary 2. If $\beta_{m} \neq 0$ and $\beta_{m} \alpha_{m} \geq 0$, then $\beta E^{h}+\alpha \bar{A}_{0}^{h}=\operatorname{diag}\left(\beta_{1} E^{h}+\right.$ $\left.\alpha_{1} \bar{A}_{10}^{h}, \ldots, \beta_{d} E^{h}+\alpha_{d} \bar{A}_{d 0}^{h}\right)$ is invertible and $\|\left(\beta E^{h}+\alpha \bar{A}_{0}^{h}\right)^{-1}|| \leq 1 / \min _{1 \leq m \leq d}\left|\beta_{m}\right|$.

Using Lemma 3, Eqs (3.5) and (3.6) are equivalent to

$$
\left(E^{h}+\left(\beta E^{h}+\alpha \bar{A}_{0}^{h}\right)^{-1}\left(\alpha \bar{A}_{1}^{h}+\alpha \bar{A}_{2}^{h}+\beta \bar{K}^{h}\right)\right) \bar{W}^{h}=\left(\beta E^{h}+\alpha \bar{A}_{0}^{h}\right)^{-1} \bar{F}^{h} .
$$

Let $S^{h_{m}}=\operatorname{span}\left\{e_{j}(t), j=1, \ldots, n_{m}\right\} \subset C[0,1]$ denote a piecewise linear function subspace with the basis points $\left\{t_{i}\right\}_{i=1}^{n_{m}}$, where $\left\{e_{j}(t), j=1, \ldots, n_{m}\right\}$ are the basis functions satisfying $e_{j}\left(t_{i}\right)=\delta_{j i}$. Define a prolongation operator $I^{h_{m}}: \Re^{n_{m}} \rightarrow S^{h_{m}}$ satisfying

$$
I^{h_{m}} v=\sum_{j=1}^{n_{m}} v_{j} e_{j}(t), \forall v=\left(v_{1}, \ldots, v_{n_{m}}\right) \in \Re^{n_{m}},
$$

and a restricted operator $R^{h_{m}}: C[0,1] \rightarrow \Re^{n_{m}}$ satisfying

$$
R^{h_{m}} v=\left(v\left(t_{1}\right), \ldots, v\left(t_{n_{m}}\right)\right) \in \Re^{n_{m}}, \forall v \in C[0,1] .
$$

We have the following lemma.

Lemma 4. If $\beta_{m}=0$, then the operator sequence, $\left\{I^{h_{m}}\left(\bar{A}_{m 0}^{h}\right)^{-1} R^{h_{m}} \bar{A}_{m 0}\right.$ : $\left.C^{3}[0,1] \rightarrow C[0,1], m=1, \ldots, d\right\}$, is uniformly bounded and convergent to the embedding operator $I$.

Proof. For any given $v \in C^{3}[0,1]$, we construct an operator equation

$$
\bar{A}_{m 0} v=f
$$

and its approximate equation: find $v^{h} \in \Re^{n_{m}}$ such that

$$
\left(\bar{A}_{m 0}^{h} v^{h}\right)\left(t_{i}\right)=f\left(t_{i}\right)\left(i=1, \ldots, n_{m}\right) .
$$

Let $e \in \Re^{n_{m}}$ with $e\left(t_{j}\right)=v^{h}\left(t_{j}\right)-v\left(t_{j}\right)\left(j=1, \ldots, n_{m}\right)$. Then we have the linear equations

$$
\bar{A}_{m 0}^{h} e=\varepsilon,
$$


where $e^{T}=\left(e\left(t_{1}\right), \ldots, e\left(t_{n_{m}}\right)\right)$ and $\varepsilon^{T}=\left(\varepsilon\left(t_{1}\right), \ldots, \varepsilon\left(t_{n_{m}}\right)\right)$. From (3.3) we obtain

$$
\begin{aligned}
\varepsilon\left(t_{i}\right)= & \int_{0}^{1} a_{m 0}\left(t_{i}, \tau\right) v(\tau) d \tau \\
& -\left[\sum_{j=1, j \neq i}^{n_{m}} h_{m} a_{m 0}\left(t_{i}, t_{j}\right) v\left(t_{j}\right)+c_{0} h_{m} \ln \left(e^{-1 / 2} / n_{m}\right) v\left(t_{i}\right)\right]=O\left(h_{m}^{3}\right) .
\end{aligned}
$$

Thus it implies that $\varepsilon \|=O\left(h_{m}^{3}\right)$ and

$$
\begin{aligned}
\|e\| & =\left\|\left(\bar{A}_{m 0}^{h}\right)^{-1} R^{h_{m}} \varepsilon\right\|=\left\|R^{h_{m}}\left(\bar{A}_{m 0}\right)^{-1} f-\left(\bar{A}_{m 0}^{h}\right)^{-1} R^{h_{m}} f\right\| \\
& =\left\|R^{h_{m}} v-\left(\bar{A}_{m 0}^{h}\right)^{-1} R^{h_{m}} A_{m 0} v\right\|=O\left(h_{m}^{2}\right) .
\end{aligned}
$$

Since $I^{h_{m}} R^{h_{m}} \rightarrow I$, the proof of Lemma 4 is completed.

Corollary 3. Let the Nystrom approximation $D^{h}$ be defined by (3.1). (1) For $\beta_{m}=0$ and $\Gamma_{q} \cap \Gamma_{m}=\emptyset$, we have

$$
I^{h_{m}}\left(\bar{A}_{m 0}^{h}\right)^{-1} R^{h_{m}} D^{h} \stackrel{c . c}{\rightarrow}\left(\bar{A}_{m 0}\right)^{-1} D, \text { in } C[0,1] \rightarrow C[0,1] .
$$

Also $E^{h}+I^{h_{m}}\left(\bar{A}_{m 0}^{h}\right)^{-1} R^{h_{m}} D^{h}$ is invertible, and its inverse operator is uniformly bounded.

(2) For $\beta_{m}=0$ and $\Gamma_{q} \cap \Gamma_{m} \neq \emptyset$, we have

$$
I^{h_{m}}\left(\bar{A}_{m 0}^{h}\right)^{-1} R^{h_{m}} \tilde{A}_{q m}^{h} \stackrel{c . c}{\rightarrow}\left(\bar{A}_{m 0}\right)^{-1} \tilde{A}_{q m}, \text { in } C[0,1] \rightarrow C[0,1] .
$$

Also $E^{h}+I^{h_{m}}\left(A_{00}^{h_{m}}\right)^{-1} R^{h_{m}} \tilde{A}_{q m}^{h}$ is invertible, and its inverse operator is uniformly bounded, where $\tilde{A}_{q m}^{h}$ is the Nyström approximation of the integral operator $\tilde{A}_{q m}$ with kernel $\tilde{a}_{q m}(t, \tau)=\sin ^{p}(\pi t) a_{q m}(t, \tau)$.

(3) For $\beta_{m} \neq 0$, we have

$$
\begin{aligned}
& I^{h_{m}}\left(\beta_{m} E^{h}+\alpha_{m} \bar{A}_{m 0}^{h}\right)^{-1} R^{h_{m}} D^{h} \stackrel{c . c}{\rightarrow}\left(\beta_{m} E+\alpha_{m} \bar{A}_{m 0}\right)^{-1} D, \\
& \quad \text { in } C[0,1] \rightarrow C[0,1] .
\end{aligned}
$$

Also $E^{h}+I^{h_{m}}\left(\beta_{m} E^{h}+\alpha_{m} \bar{A}_{m 0}^{h}\right)^{-1} R^{h_{m}} D^{h}$ is invertible, and its inverse operator is uniformly bounded.

Proof. If $\beta_{m}=0$ and $\Gamma_{q} \cap \Gamma_{m}=\emptyset$, obviously, the kernel $d(t, \tau)$ of the operator $D$ and its derivatives of higher order are continuous $[5,11,15,21,25]$. Since

$$
\left\|I^{h_{m}}\left(\bar{A}_{m 0}^{h}\right)^{-1} R^{h_{m}} D^{h}\right\|_{0,0} \leq\left\|I^{h_{m}}\left(\bar{A}_{m 0}^{h}\right)^{-1} R^{h_{m}} \bar{A}_{m 0}\right\|_{0,3}\left\|\left(\bar{A}_{m 0}\right)^{-1} D^{h}\right\|_{3,0}
$$


by Lemma 4 and $\left(\bar{A}_{m 0}\right)^{-1} D^{h} \in \mathcal{L}\left(C[0,1], C^{3}[0,1]\right)$, there exists a constant $c$ such that

$$
\left\|\left(\bar{A}_{m 0}\right)^{-1} D^{h}\right\|_{3,0} \leq c, \text { and }\left\|I^{h_{m}}\left(\bar{A}_{m 0}^{h}\right)^{-1} R^{h_{m}} \bar{A}_{m 0}\right\|_{0,3} \leq c
$$

where $\|\cdot\|_{m_{2}, m_{1}}$ is the norm of the linear bounded operator space $\mathcal{L}\left(C^{m_{1}}[0,1]\right.$, $\left.C^{m_{2}}[0,1]\right)$. Using the results of $[3,6,12]$, the operator sequence $\left\{\left(\bar{A}_{m 0}\right)^{-1} D^{h}\right.$ : $\left.C[0,1] \rightarrow C^{3}[0,1]\right\}$ must be collectively compact convergent to $\left(\bar{A}_{m 0}\right)^{-1} D$. Hence, we complete the proof of (1). Similarly, we can prove (2) and (3).

Define the subspace

$$
C_{0}[0,1]=\left\{v(t) \in C[0,1]: v(t) / \sin ^{3}(\pi t) \in C[0,1]\right\}
$$

of the space $C[0,1]$ with the norm $\|v\|^{*}=\max _{0 \leq t \leq 1}\left|v(t) / \sin ^{3}(\pi t)\right|$. Replacing $\left(M^{h}\right)^{-1}=\left(\beta E^{h}+\alpha \bar{A}_{0}^{h}\right)^{-1}, \alpha \bar{A}_{1}^{h}, \alpha \bar{A}_{2}^{h}$, and $\beta \bar{K}^{h}$ by $\left(\hat{M}^{h}\right)^{-1}=I^{h}\left(M^{h}\right)^{-1} R^{h}$, $\hat{A}_{1}^{h}=I^{h} \alpha \bar{A}_{1}^{h} R^{h}, \hat{A}_{2}^{h}=I^{h} \alpha \bar{A}_{2}^{h} R^{h}$, and $\hat{K}^{h}=I^{h} \beta \bar{K}^{h} R^{h}$, respectively, we obtain the operator

$$
\hat{L}^{h}=I^{h}\left(M^{h}\right)^{-1} R^{h}\left(\alpha \bar{A}_{1}^{h}+\alpha \bar{A}_{2}^{h}+\beta \bar{K}^{h}\right) R^{h},
$$

mapping $\mathcal{V}$ to $\prod_{m=1}^{d} S^{h_{m}}$, where $R^{h}=\operatorname{diag}\left(R^{h_{1}}, \ldots, R^{h_{d}}\right), I^{h}=\operatorname{diag}\left(I^{h_{1}}, \ldots, I^{h_{d}}\right)$, and

$$
\mathcal{V}= \begin{cases}(C[0,1])^{d}, & \text { as } \beta_{m} \neq 0, m=1, \ldots, d \\ (C[0,1])^{k} \times\left(C_{0}[0,1]\right)^{d-k}, & \text { as } \beta_{i_{m}} \neq 0, m=1, \ldots, k, i_{m} \in\{1, \ldots, d\}, \\ \left(C_{0}[0,1]\right)^{d}, & \text { as } \beta_{m}=0, m=1, \ldots, d .\end{cases}
$$

Now consider the operator equation

$$
\left(E^{h}+\hat{L}^{h}\right) \hat{W}^{h}=\hat{F}^{h}
$$

with $\hat{F}^{h}=I^{h}\left(\beta E^{h}+\alpha \bar{A}_{0}^{h}\right)^{-1} R^{h} \bar{F}^{h}$. Obviously, if $\hat{W}^{h}=I^{h} \bar{W}^{h}$ is a solution of (3.21), then $R^{h} \hat{W}^{h}$ must be a solution of (3.15); conversely, if $\bar{W}^{h}$ is a solution of (3.15), then $I^{h} \bar{W}^{h}$ must be a solution of (3.21). Below we prove that there exists a unique solution $\hat{W}^{h}$ in (3.21) such that $\hat{W}^{h}$ converges to $\bar{W}$.

Theorem 1. The operator sequence $\left\{\hat{L}^{h}\right\}$ is collectively compact convergent to $L=\left(\beta E+\alpha \bar{A}_{0}\right)^{-1}\left(\alpha \bar{A}_{1}+\alpha \bar{A}_{2}+\beta \bar{K}\right)$ in $\mathcal{V}$, i.e.,

$$
\hat{L}^{h} \stackrel{c . c}{\rightarrow} L .
$$

Proof. Let $\Theta=\{v:\|v\| \leq 1, v \in \mathcal{V}\}$ be a unit ball, and $H=\left\{H^{(1)}, H^{(2)}, \ldots\right\}$ be a mesh sequence, where $H^{(n)}=\left\{h_{1}^{(n)}, \ldots, h_{d}^{(n)}\right\}$ denotes a multi-parameter step 
size with $\max _{1 \leq m \leq d} h_{m}^{(n)} \rightarrow 0$ as $n_{m} \rightarrow \infty$. For an arbitrary sequence $\left\{Z_{h}=\right.$ $\left.\left(Z_{1}^{h}, \ldots, Z_{d}^{h}\right): h \in H\right\} \subset \Theta$ satisfying

$$
\max _{1 \leq m \leq d, 0 \leq t \leq 1}\left|Z_{m}^{h}(t) / \sin ^{3}(\pi t)\right| \leq 1,
$$

we conclude that there exists a convergent subsequence in $\left\{\hat{L}^{h} Z_{h}\right\}$. Firstly, for $\beta_{q}=0(q=1, \ldots, d)$, since

$$
\begin{aligned}
& \left\|I^{h_{1}}\left(\bar{A}_{10}^{h}\right)^{-1} R^{h_{m}} A_{12}^{h}\right\|_{0}=\left\|I^{h_{1}}\left(\bar{A}_{00}^{h}\right)^{-1} R^{h_{m}} \tilde{A}_{12}^{h}\left(Z_{m}^{h}\right) / \sin ^{3}(\pi t)\right\|_{0} \\
& \quad \leq\left\|I^{h_{1}}\left(\bar{A}_{10}^{h}\right)^{-1} R^{h_{m}} \bar{A}_{10}\right\|_{0,3}\left\|\bar{A}_{10}^{-1} \tilde{A}_{12}^{h}\right\|_{3,0}\left\|Z_{m}^{h}\right\|^{*},
\end{aligned}
$$

from Corollary 3 we can prove that there exists a convergent subsequence in

$$
\left\{I^{h_{1}}\left(\bar{A}_{10}^{h}\right)^{-1} R^{h_{m}} A_{12}^{h} Z_{m}^{h}\right\} \subset C_{0}[0,1] \subset C[0,1]
$$

such that

$I^{h_{1}}\left(\bar{A}_{10}^{h}\right)^{-1} R^{h_{m}} \bar{A}_{1 m}^{h} \stackrel{c . c}{\rightarrow}\left(\bar{A}_{10}\right)^{-1} \bar{A}_{1 m}$, in $\mathcal{L}(C[0,1], C[0,1])$, for $2<m \leq d$,

and

$$
I^{h_{1}}\left(\bar{A}_{10}^{h}\right)^{-1} R^{h_{m}} \bar{A}_{01}^{h} \stackrel{c . c}{\rightarrow}\left(\bar{A}_{10}\right)^{-1} \bar{A}_{01}, \text { in } \mathcal{L}(C[0,1], C[0,1]) .
$$

Also since $C_{0}[0,1] \subset C[0,1]$, we can find an infinite subsequence $H_{1} \subset H$ such that the first component of $\hat{L}^{h} Z_{h}$,

$$
\sum_{m=1}^{d} I^{h_{1}}\left(\beta_{1} E^{h_{1}}+\alpha_{1} \bar{A}_{10}^{h}\right)^{-1} R^{h_{1}}\left(\alpha_{1} \bar{A}_{01}^{h}+\alpha_{1} \bar{A}_{1 m}^{h}+\beta_{1} \bar{K}_{1 m}^{h}\right) R^{h_{1}} Z_{m}^{h}
$$

converges as $h \rightarrow 0$, where $h \in H_{1}$ (see $[3,6,11,12]$ ). Based on the above methods, we may find a subsequence, $H_{d} \subset H_{d-1} \subset \ldots \subset H_{1} \subset H$, such that $\left\{\hat{L}^{h} Z_{h}, h \in H_{d}\right\}$ is a convergent sequence in $\mathcal{V}$, which implies

$$
\hat{L}^{h} \stackrel{p}{\rightarrow} L
$$

where the notation $\stackrel{p}{\rightarrow}$ denotes the pointwise convergence. From [3, 6, 11, 12], we conclude that $\hat{L}^{h} \stackrel{c . c}{\rightarrow} L$ in $\mathcal{V}$.

Secondly, if $\beta_{q} \neq 0(q=1, \ldots, d)$, from Corollary 3 we obtain

$$
\begin{aligned}
& I^{h_{1}}\left(\beta_{q} E^{h_{q}}+\alpha_{q} \bar{A}_{q 0}^{h}\right)^{-1} R^{h_{q}} \alpha_{q} \bar{A}_{0 q}^{h} \stackrel{c . c}{\rightarrow}\left(\beta_{q} E+\alpha_{q} \bar{A}_{q 0}\right)^{-1} \alpha_{q} \bar{A}_{0 q}, \\
& I^{h_{q}}\left(\beta_{q} E^{h_{q}}+\alpha_{q} \bar{A}_{q 0}^{h}\right)^{-1} R^{h_{q}} \alpha_{q} \bar{A}_{q m}^{h} \stackrel{c . c}{\rightarrow}\left(\beta_{q} E+\alpha_{q} \bar{A}_{q 0}\right)^{-1} \alpha_{q} \bar{A}_{q m}
\end{aligned}
$$


and

$$
I^{h_{q}}\left(\beta_{q} E^{h_{q}}+\alpha_{q} \bar{A}_{q 0}^{h}\right)^{-1} R^{h_{q}} \beta_{q} \bar{K}_{q m}^{h} \stackrel{c . c}{\rightarrow}\left(\beta_{q} E+\alpha_{q} \bar{A}_{q 0}\right)^{-1} \beta_{q} \bar{K}_{q m} .
$$

Using the diagonal process, we can also find a subsequence $H_{d} \subset H$ such that (3.25) converges as $h \rightarrow 0$. Thus we conclude $\hat{L}^{h} \stackrel{c . c}{\rightarrow} L$ in $\mathcal{V}$, too.

Finally, if $\beta_{i_{m}} \neq 0\left(m=1, \ldots, k, i_{m} \in\{1, \ldots, d\}\right)$, the proof of $\hat{L}^{h} \stackrel{c . c}{\rightarrow} L$ in $\mathcal{V}$ is similar.

From Theorem 1 we have the following corollary.

Corollary 4. ([3, 12]). Assume that (1.2) has a unique solution and $h=$ $\max _{1 \leq m \leq d} h_{m}$ is sufficiently small, then there exists a unique solution $\hat{W}^{h}$ in (3.21), and $\hat{W}^{h}$ has the following error bound under the norm of $\mathcal{V}$,

$$
\left\|\hat{W}^{h}-\bar{W}\right\| \leq\left\|(I+L)^{-1}\right\| \frac{\left\|\left(\hat{L}^{h}-L\right) \hat{F}\right\|+\left\|\left(\hat{L}^{h}-L\right) \hat{L}^{h} \bar{W}\right\|}{1-\left\|\left(I+\hat{L}^{h}\right)^{-1}\left(\hat{L}^{h}-L\right) \hat{L}^{h}\right\|} .
$$

\section{Multivariate Asymptotic Expansions of Errors and SEM}

In the section, we shall derive the multi-parameter asymptotic expansions of the solution errors, and describe the algorithm of the SEM.

Theorem 2. Assume that there exists a unique solution in (1.2), $F^{h}$ is computed by (3.1) and (3.3), and $\bar{x}_{m i}(t), \bar{g}_{m}(t) \in \bar{C}^{4}[0,1](i=1,2, m=1, \ldots, d)$. Then there exists the vector function $\varpi=\left(\varpi_{1}, \ldots, \varpi_{d}\right)^{T} \in \mathcal{V}$ independent of $h=\left(h_{1}, \ldots, h_{d}\right)^{T}$ such that

$$
\left.\left(\bar{W}-\hat{W}^{h}\right)\right|_{t=t_{j}}=\left.\operatorname{diag}\left(h_{1}^{3}, \ldots, h_{d}^{3}\right) \varpi\right|_{t=t_{j}}+O\left(h^{4}\right), h=\max _{1 \leq m \leq d} h_{m} .
$$

Proof. By the quadrature rule (3.1) and (3.3), there exists the asymptotic expansion $[8,20]$

$$
\left.\left(\bar{F}-\hat{F}^{h}\right)\right|_{t=t_{i}}=\left.\operatorname{diag}\left(h_{1}^{3}, \ldots, h_{d}^{3}\right) I^{h} R^{h} v\right|_{t=t_{i}}+O\left(h^{4}\right),
$$

where $h=\max _{m=1}^{d} h_{m}$ and $v=\left(v_{1}, \ldots, v_{d}\right)^{T}$ with $v_{m}=-\eta_{m} \xi^{\prime}(-2)\left(z_{m}(t) \bar{g}_{m}(t)\right)^{\prime \prime}$ $/ \pi$ and

$$
\eta_{m}=\left\{\begin{array}{c}
0, \text { as } \beta_{m}=0 \\
1, \operatorname{as} \beta_{m} \neq 0 .
\end{array}\right.
$$


Using (2.11), (3.2), (3.4), (3.5) and (3.6), we can easily obtain

$$
\begin{aligned}
& \left.\left(\beta E^{h}+\alpha \bar{A}_{0}^{h}+\alpha \bar{A}_{1}^{h}+\alpha \bar{A}_{2}^{h}+\beta \bar{K}^{h}\right) R^{h}\left(\hat{W}^{h}-\bar{W}\right)\right|_{t=t_{i}} \\
= & \hat{F}^{h}-\left.I^{h}\left(\beta E^{h}+\alpha \bar{A}_{0}^{h}+\alpha \bar{A}_{1}^{h}+\alpha \bar{A}_{2}^{h}+\beta \bar{K}^{h}\right) R^{h} \bar{W}\right|_{t=t_{i}} \\
= & \hat{F}^{h}-\left[\left(\left(\beta E+\alpha \bar{A}_{0}+\alpha \bar{A}_{1}+\alpha \bar{A}_{2}+\beta \bar{K}\right) \bar{W}\right.\right. \\
& \left.\left.-\operatorname{diag}\left(h_{1}^{3}, \ldots, h_{d}^{3}\right) I^{h} R^{h} \gamma\right)\left.\right|_{t=t_{i}}+O\left(h^{4}\right)\right] \\
= & \left.\left(\hat{F}^{h}-F\right)\right|_{t=t_{i}}+\left.\operatorname{diag}\left(h_{1}^{3}, \ldots, h_{d}^{3}\right) I^{h} R^{h} \gamma\right|_{t=t_{i}}+O\left(h^{4}\right) \\
= & \left.\operatorname{diag}\left(h_{1}^{3}, \ldots, h_{d}^{3}\right) I^{h} R^{h} \varphi\right|_{t=t_{i}}+O\left(h^{4}\right), h=\max _{1 \leq m \leq d} h_{m},
\end{aligned}
$$

where $\gamma=\left(\gamma_{1}, \ldots, \gamma_{d}\right)^{T}$ with $\gamma_{m}=\alpha_{m} \xi^{\prime}(-2)\left(z_{p}(t) \bar{w}_{m}(t)\right)^{\prime \prime} / \pi$, and $\varphi=\left(\varphi_{1}, \ldots\right.$, $\left.\varphi_{d}\right)^{T}$ with $\varphi_{m}=v_{m}+\gamma_{m}$. From Corollary 3 we get

(4.3) $\left.\left.\left(E^{h}+\hat{L}_{h}\right)\left(\bar{W}-\hat{W}^{h}\right)\right|_{t=t_{i}}=\operatorname{diag}\left(h_{1}^{3}, \ldots, h_{d}^{3}\right)\left(\hat{M}_{1}^{h}\right)^{-1} I^{h} R^{h} \varphi\right)\left.\right|_{t=t_{i}}+O\left(h^{4}\right)$.

Define the auxiliary equation

$$
(E+L) \varpi=M^{-1} \varphi
$$

and its approximate equation

$$
\left(E^{h}+\hat{L}^{h}\right) \varpi^{h}=\left(\hat{M}^{h}\right)^{-1} I^{h} R^{h} \varphi .
$$

Substituting (4.4) and (4.5) into (4.3) yields

$$
\left.\left(E^{h}+\hat{L}^{h}\right)\left(\bar{W}-\hat{W}^{h}-\operatorname{diag}\left(h_{1}^{3}, \ldots, h_{d}^{3}\right) \varpi^{h}\right)\right|_{t=t_{i}}=O\left(h^{4}\right), h=\max _{1 \leq m \leq d} h_{m} .
$$

Since $\left(E^{h}+\hat{L}^{h}\right)^{-1}$ is uniformly bounded from Theorem 1, we get

$$
\left.\left(\bar{W}-\hat{W}^{h}-\operatorname{diag}\left(h_{1}^{3}, \ldots, h_{d}^{3}\right) \varpi^{h}\right)\right|_{t=t_{i}}=O\left(h^{4}\right), h=\max _{1 \leq m \leq d} h_{m} .
$$

Replacing $\varpi^{h}$ in (4.6) by $\varpi$ and applying (3.26), we complete the proof of Theorem 2.

The multi-parameter asymptotic expansions (4.1) imply that SEM can be applied to solve (1.2). Moreover, the high order $O\left(h^{4}\right)$ of accuracy can be obtained by computing some coarse grids of $\Gamma_{m}(m=1, \ldots, d)$ in parallel. The algorithms of the SEM are described as follows $[11,13]$ :

Step 1. Take $h^{(0)}=\left(h_{1}, \ldots, h_{d}\right)$ and $h^{(m)}=\left(h_{1}, \ldots, h_{m} / 2, \ldots, h_{d}\right)$, and solve (3.6) under mesh parameters $h^{(m)}$ in parallel, where $\bar{W}^{h^{(m)}}\left(t_{i}\right), t_{i}=(i-1 / 2) h_{m}$ $\left(i=1, . ., n_{m}, m=1, \ldots, d\right)$ denote their solutions. 
Step 2. Compute for the solutions at the coarse grid points

$$
\bar{W}^{*}\left(t_{i}\right)=8 / 7\left[\sum_{m=1}^{d} \bar{W}^{h^{(m)}}\left(t_{i}\right)-(d-7 / 8) \bar{W}^{h^{(0)}}\left(t_{i}\right)\right],
$$

and $u(y)(y \in \Omega)$ can then be obtained by (3.7).

Moreover, using $\left|\bar{W}^{*}\left(t_{i}\right)-\bar{W}\left(t_{i}\right)\right|=O\left(h^{4}\right)$, we obtain a posteriori estimates

$$
\begin{aligned}
& \left|\bar{W}\left(t_{i}\right)-\frac{1}{d} \sum_{m=1}^{d} \bar{W}^{h^{(m)}}\left(t_{i}\right)\right| \\
\leq & \left|\bar{W}\left(t_{i}\right)-\frac{8}{7}\left[\sum_{m=1}^{d} \bar{W}^{h^{(m)}}\left(t_{i}\right)-\left(d-\frac{7}{8}\right) \bar{W}^{h^{(0)}}\left(t_{i}\right)\right]\right| \\
& +\left(\frac{8 d}{7}-1\right)\left|\frac{1}{d} \sum_{m=1}^{d} \bar{W}^{h^{(m)}}\left(t_{i}\right)-\bar{W}^{h^{(0)}}\left(t_{i}\right)\right| \\
\leq & \left(\frac{8 d}{7}-1\right)\left|\frac{1}{d} \sum_{m=1}^{d} \bar{W}^{h^{(m)}}\left(t_{i}\right)-\bar{W}^{h^{(0)}}\left(t_{i}\right)\right|+O\left(h^{4}\right) .
\end{aligned}
$$

\section{NUMERICAL EXAMPLES}

Consider the problem (1.1) in the first quadrant of the unit disc,

$$
\Gamma: x_{1}=0, x_{2}=0 \text { and } x_{1}^{2}+x_{2}^{2}=1, x_{1}>0, x_{2}>0,
$$

with the mixed boundary conditions ${ }^{[4]}$

$$
\begin{cases}u=1 & \text { on the circular, } \\ u=0 & \text { on } x_{2}=0 \\ \frac{\partial u}{\partial n}=0 & \text { on } x_{1}=0\end{cases}
$$

This problem has the analytic solution: $u=2 / \pi \arctan \left(2 x_{2} /\left(1-x_{1}^{2}-x_{2}^{2}\right)\right)$. Using $\varphi_{4}(t)$ in (2.4)-(2.6), Table 1 lists the errors $e_{p}=\left|u(P)-u^{h}(P)\right|$, the SEM-errors, and the error rates $r_{P}=\left|\left(u(P)-u^{h}(P)\right) /\left(u(P)-u^{2 h}(P)\right)\right|$ with $P=A(0.1,0.1)$ or $B(0.9,0.1)$ by MQM and SEM, where

$$
\mathrm{SEM}-\text { error }=\left|u(P)-8 / 7\left[\sum_{m=1}^{d} u^{h^{(m)}}(P)-(d-7 / 8) u^{h^{(0)}}(P)\right]\right|,
$$

and $u^{h^{(m)}}(P)$ is computed by (3.7). Moreover, in Table 1 we also list the a posteriori estimates with (a-post-error) $=\left(\frac{8 d}{7}-1\right)\left|\frac{1}{d} \sum_{m=1}^{d} u^{h^{(m)}}(P)-u^{h^{(0)}}(P)\right|$, and average 
errors with (average-error) $=\left|u(P)-\frac{1}{d} \sum_{m=1}^{d} u^{h^{(m)}}(P)\right|$, where $\left(n_{1}, n_{2}, n_{3}\right)$ denotes the numbers of nodes on three edges of $\Gamma$. When $n=64$, the numerical results are given by $e_{A}=1.00 E-3$ and $e_{B}=1.20 E-3$ in [4]. For Table 1 , when $\Sigma_{m=1}^{3} n_{m}=$ 32 , the SEM errors for $\left(n_{1}, n_{2}, n_{3}\right)=(8,8,8)$ are given by $e_{A}=1.23 E-4$ and $e_{B}=4.732 E-4$, which are more accurate.

From Table 1, we can see $r_{A}$ and $r_{B} \approx 8$, to indicate the $O\left(h^{3}\right)$ convergence rate for the MQM, and $r_{A}$ and $r_{B} \approx 16$ to indicate the $O\left(h^{4}\right)$ convergence rate for the SEM. Those results coincide with the theoretical analysis made.

In summary, the MQM and the SEM have the $O\left(h^{3}\right)$ and the $O\left(h^{4}\right)$ convergence rates, respectively. Moreover, their algorithms are simple and easy to carry out. Hence, the MQM and the SEM are more advantageous over the Galerkin and the collocation methods, and they are recommended for solving the BIE.

Table 1. The errors, the a posteriori errors and SEM-errors

\begin{tabular}{|c|c|c|c|c|c|c|c|}
\hline$\left(n_{1}, n_{2}, n_{3}\right)$ & $e_{A}$ & $e_{B}$ & $\left(n_{1}, n_{2}, n_{3}\right)$ & $e_{A}$ & $r_{A}$ & $e_{B}$ & $r_{B}$ \\
\hline$(4,4,4)$ & $2.324(-2)$ & $7.352(-2)$ & $(8,8,8)$ & $3.653(-3)$ & 6.4 & $9.815(-3)$ & 7.5 \\
\hline$(8,4,4)$ & $1.830(-2)$ & $5.193(-2)$ & $(16,8,8)$ & $2.407(-3)$ & 7.6 & $6.271(-3)$ & 8.3 \\
\hline$(4,8,4)$ & $1.444(-2)$ & $3.828(-2)$ & $(8,16,8)$ & $2.005(-3)$ & 7.2 & $5.182(-3)$ & 7.4 \\
\hline$(4,4,8)$ & $1.483(-2)$ & $4.546(-2)$ & $(8,8,16)$ & $2.088(-3)$ & 7.1 & $6.131(-3)$ & 7.3 \\
\hline average-error & $1.585(-2)$ & $4.522(-2)$ & average-error & $2.166(-3)$ & 7.3 & $5.861(-3)$ & 7.7 \\
\hline a-post-error & $1.793(-2)$ & $5.559(-2)$ & a-post-error & $2.974(-3)$ & 6.0 & $7.907(-3)$ & 7.0 \\
\hline SEM-error & $2.074(-3)$ & $8.011(-3)$ & SEM-error & $1.234(-4)$ & 16.8 & $4.732(-4)$ & 16.9 \\
\hline
\end{tabular}

\section{ACKNOWLEDGMENT}

We are grant grateful for W. W. Lin and the referee for their valuable comments and suggestions.

\section{REFERENCES}

1. K. Atkinson, The numerical solution of Laplace's equation on a wedge, IMA. J Num. Anal., 4 (1984), 19-41.

2. K. E. Atkinson and G. Chandler, Boundary integral equation methods for solving Laplace's equation with nonlinear boundary conditions: The smooth boundary case, Math. Comp., 55 (1990), 451-472.

3. P. M. Anselone, Collectively Compact Operator Approximation Theory, PrenticeHall, Englewood Cliffs, New Jersey, 1971.

4. C. A. Brebbia, Boundary Element Methods, Butterworths, 1980.

5. G. Chandler, Galerkin's method for boundary integral equations on polygonal domains, Aust. Math Soc. Ser., 26 (1984), 1-13. 
6. F. Chatelin, Spectral Approximation of Linear Operator, Academic Press, 1983.

7. Z. Daniel, Standard Mathematical Tables and Formulae, New York, 1996.

8. P. Davis, Methods of Numerical Integration, Second, Academic Press, New York, 1984.

9. D. L. Dwoyer, M. Y. Hussaini and R. G. Voigt, Finite Elements Theory and Application, Springer-Verlag, New York, 1986.

10. A. S. Householder, The Theory of Matrices in Numerical Analysis, Blaisdell Publishing Company, 1964.

11. J. Huang and T. Lü, The mechanical quadrature methods and their splitting extrapolations for solving first-kind boundary integral equations on polygonal regions (in Chinese), Math. Num. Sinica, 1 (2004), 51-60.

12. R. Kress, Linear Integral Equations, Springer-Verlag, 1989.

13. C. B. Lin, T. Lü and T. M. Shih, The Splitting Extrapolation Method, World Scientific, Singapore, 1995.

14. Q. Lin and T. Lü, Splitting extrapolation for multidimensional problem, J. Comp. Math., 1 (1983), 376-383.

15. T. Lü and J. Huang, Quadrature methods with high accuracy and extrapolation for solving boundary integral equations of first-kind (in Chinese), Math Num. Sinica, 1 (2000), 59-72.

16. T. Lü and J. Lu, Splitting extrapolation for solving the second order elliptic system with curved boundary in $\mathrm{R}^{d}$ by using d-quadratic isoparametric finite element, Appl. Numer. Math., 40 (2002), 467-481.

17. F. Paris and J. Cans, Boundary Element Method, Oxford University Press, 1997.

18. U. Rüde and A. Zhou, Multi-parameter extrapolation methods for boundary integral equations, Advances in Computational Mathematics, 9 (1998), 173-190.

19. K. Ruotsalainen and W. W Endland, On the boundary element method for some nonlinear boundary value problems, Numer. Math., 53 (1988), 299-314.

20. A. Sidi and M. Israrli, Quadrature methods for periodic singular Fredholm integral equation, J. of Sci Comp., 3 (1988), 201-231.

21. A. Sidi, A new variable transformation for numerical integration, I. S., Num Math., 112 (1993), 359-373.

22. I. H. Sloan and A. Spence, The Galerkin method for integral equations of first-kind with logarithmic kernel, IMA J. Numer. Anal., V (1988), 105-122.

23. J. H. Wilkinson, The Algebraic Eigenvalue Problem, Oxford University Press, 1965.

24. Y. S. Xu and Y. H. Zhao, An extrapolation method for a class of boundary integral equations, Math. Comp., 65 (1996), 587-610. 
25. Y. Yi, The collocation method for first-kind boundary integral equations on polygonal regions, Math Comp., 189 (1990), 139-154.

Jin Huang

College of Applied Mathematics,

University of Electronic and Science Technology of China,

Chengdu, China

E-mail: huangjin12345@163.com

Zi Cai Li

Department of Applied Mathematics,

and Department of Computer Science and Engineering,

National Sun Yat-sen University,

Kaohsiung, Taiwan

E-mail: zcli@math.nsysu.edu.tw

Tao Lü and Rui Zhu

Mathematical College,

Sichuan University,

Chengdu, China 